\title{
School readiness and academic achievement of children with hearing impairment: A South African exploratory study
}

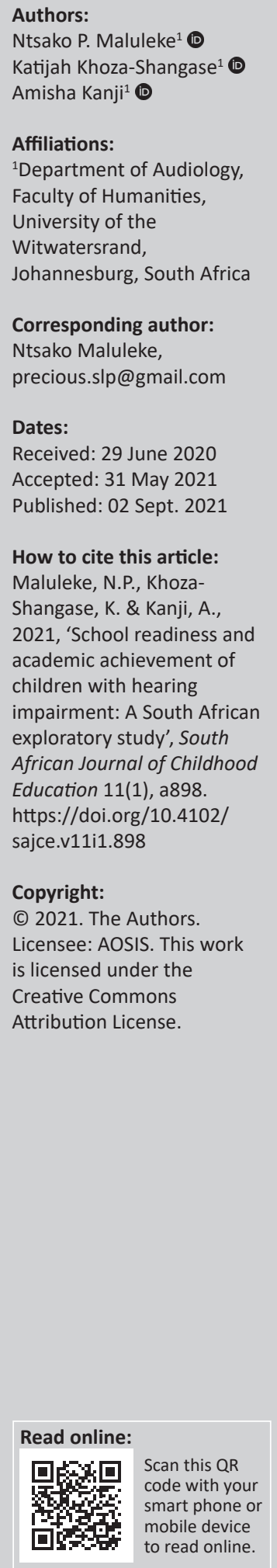

Authors:

Katijah Khoza-Shangase ${ }^{1}$

Affiliations:

Department of Audiology,

University of the

Witwatersrand,

Ntsako Maluleke,

Dates:

Accepted: 31 May 2021

How to cite this article:

Maluleke, N.P., Khoza-

academic achievement of

children with hearing

African Journal of Childhood

Education 11(1), a898.

https://doi.org/10.4102/

(0)

Licensee: AOSIS. This work

is licensed under the

Creative Commons

mobile device

to read online.
Background: Children with hearing impairment may be at risk of not achieving the necessary school readiness because of the link between hearing impairment and academic achievement. However, early hearing detection and intervention (EHDI) can improve potential outcomes for these children.

Aim: As part of a bigger study titled 'Communication and school readiness abilities of hearingimpaired preschool graduates: Exploring outcomes of early intervention preschool programs in Gauteng', the aim of this study was to describe the school readiness and academic achievement of children with hearing impairment through retrospective record reviews of EHDI preschool records and Grade 3 teachers' survey.

Setting: The study was conducted in Gauteng, South Africa, where two EHDI preschool centres participated in the study.

Methods: Eight children identified with hearing impairment and enrolled in EHDI preschools were included in the study, along with their Grade 3 teachers. Data collection was conducted through a self-developed teacher questionnaire and record reviews of the children's preschool files. Data were analysed using descriptive statistics.

Results: Three children $(n=8)$ obtained age-appropriate school readiness results and were subsequently enrolled in mainstream schools. Five children $(n=8)$ did not exhibit ageappropriate school readiness and were subsequently enrolled in remedial schools and schools for Learners with Special Education Needs (LSEN).

Conclusion: These preliminary findings demonstrate that through EHDI, children with hearing impairment are allowed to develop the school readiness required to experience academic success. Factors influencing outcomes within the South African context need to be explored in order for South Africa to benefit maximally from EHDI initiatives.

Keywords: hearing impairment; school readiness; academic achievement; early hearing detection and intervention; early intervention; schooling.

\section{Introduction}

School readiness refers to a child's developmental, emotional and social readiness to enter a new world of learning that is different from their social environment at home or preschool learning (Umat et al. 2018). It constitutes a solid foundation in early spoken language abilities, early literacy, attention skills and mathematical concepts among other factors (Harrington, Desjardin \& Shea 2010; Mukani, Ling \& Ghani 2007). The concept of school readiness has undergone major shifts during the past four decades; it has moved from a primarily maturational and/or psychobiological model of development to a more socially constructed, developmental systems approach to understanding school readiness (McClellan, Geldhof, Cameron \& Wanless 2015; National Institute of Medicine and National Research Council 2015; Marti, Merz, Repka, Landers, Noble \& Duch 2018). Thus, in addition to cognitive skills, as well as acquired knowledge and skills such as spoken language and early literacy, parental involvement and/or family experiences are crucial in preparing these children for school readiness (Harrington et al. 2010; Mukani et al. 2007; Umat et al. 2018). Furthermore, the school environment and practices must foster and support a smooth transition for children into primary school and promote learning for all children (Britto et al. 2012).

School readiness is thought to provide a foundation to support ongoing engagement in learning throughout schooling and has been linked with improved academic achievement in primary and secondary school in terms of both equity and performance (Britto et al. 2008; Darling-Hammond 
et al. 2020; Medina \& Suthers 2008). School readiness is viewed as a strategy for economic development with cognisance of the fact that human capital, the inception of which begins in the early years, is a key conduit for sustainable and viable development (Brown 2016). Thus, it is gaining currency as a viable strategy to close the learning gap and improve equity in achieving lifelong learning and optimal developmental potential among young children, especially for those who are vulnerable and disadvantaged (Britto et al. 2008).

The population of vulnerable and disadvantaged children includes those with hearing impairment (Maluleke, KhozaShangase \& Kanji 2021). These children are particularly vulnerable as they may be at risk for not achieving the necessary school readiness because of the link between hearing impairment and academic achievement (Shaver et al. 2014). Hearing ability is the foundation of spoken language development, communication and learning (Khoza-Shangase \& Kanji 2021). In a study on risk factors and school readiness consequences in late talkers, Hammer et al. (2017) found that children with stronger language abilities have better reading comprehension and decoding skills that support reading abilities. These authors also found that larger vocabulary inventories are associated with improved number naming abilities, knowledge of measurement, shapes and geometry and the ability to manipulate symbolic representation (Hammer et al. 2017). Furthermore, children with better expressive language abilities are able to communicate better with their teachers and peers and consequently, be able to regulate their behaviour and emotions (Cole, Armstrong \& Pemberton 2010; Menting, Van Lier \& Koot 2010). Thus an unidentified or lateidentified hearing impairment causes delays in language development, and those delays in turn lead to learning problems, often resulting in poor academic achievement (Packer 2018; Lang-Roth 2014). However, early identification of congenital and early-onset hearing impairment and timeous intervention can improve potential outcomes for these children, including linguistic, psycho-emotional, as well as scholastic outcomes (Maluleke, Khoza-Shangase \& Kanji 2019a; Khoza-Shangase \& Kanji 2021).

Early identification of congenital and early-onset hearing impairment and timeous intervention can be achieved through early childhood development (ECD) initiatives such as Early Hearing Detection and Intervention (EHDI) programmes. Early Hearing Detection and Intervention programmes in high-income contexts have demonstrated a reduction in the age of a confirmed hearing impairment between 2 and 3 months of age through universal newborn hearing screening services (Swanepoel 2009). The earlier a hearing impairment is identified and intervention provided, the higher the chances of reducing or minimising its negative effects, allowing children with hearing impairment the opportunity to develop to their full potential (EHDI 2018; Khoza-Shangase \& Kanji 2021; Moeller \& Tomblin 2015;
Umat et al. 2018). This can ultimately result in improved school readiness, as well as more positive academic, social and employment outcomes (Darling-Hammond et al. 2020; Moreno 2013).

The documented positive influence of EHDI programmes on future academic success highlights the importance of laying a solid foundation aimed at addressing the needs of children with hearing impairment and ensuring adequate development and school readiness for them within the South African context (Maluleke et al. 2019a; Storbeck \& Moodley 2011). These programmes would also lessen the drain on national resources by reducing school-grade repetitions, need for specialised schooling, as well as reducing the need for specialised employment opportunities that are characteristic of children with an unidentified or late-identified hearing impairment (De Villiers 2010; Shaver et al. 2014; Tinajero 2010). However, there is a significant gap between the evidence base that supports EHDI and its implementation, as illustrated by limited access and poor quality ECD within the South African context (Khoza-Shangase 2021).

South African policies ${ }^{1}$ and the National Development Plan recognise the state's responsibility for children's development by emphasising the need for an effective and integrated system to ensure that essential ECD services are accessible to all, especially those children whose development is most at risk (Viviers, Biersteker \& Moruane 2013). However, access to ECD services without similar emphasis on ensuring that such services lead to successful outcomes for these children is problematic and renders such access unproductive and fruitless expenditure for all stakeholders involved, particularly for learners with barriers to learning, such as learners with hearing impairment (Khoza-Shangase, Sebothoma \& Moroe et al. 2021). Efforts to facilitate successful outcomes include access to therapeutic services that address barriers to learning, through ECD programmes. Unfortunately, only a handful of such programmes in South Africa cater to the needs of children with hearing impairment (Khoza-Shangase 2021; Swanepoel 2009). Moreover, locally relevant evidence demonstrating the outcomes of these programmes for children with hearing impairment is lacking in South Africa (Kanji \& Khoza-Shangase 2021; Moodley \& Storbeck 2015). To our knowledge, no research has described the academic achievement of children with hearing impairment within the South African context.

To address the dearth of available contextually relevant evidence within the South African context, a descriptive research design, employing a retrospective record review of EHDI preschool records and self-administered teacher questionnaires was conducted in order to address the study's objectives. This was part of a bigger study titled 'Communication and school readiness abilities of hearingimpaired preschool graduates: Exploring outcomes of early

1.The policies include the White Paper on Education and Training (Department of Education 1995), the White Paper for Social Welfare (Department of Welfare 1997), Educa the White Paper for the Trans), the Education Whit Paptem in South Africa (Department of Welfare 1997), the Education White Paper 5 on Early Childhood Education (Department of Education 2001) and the National Integrated Plan for Early Childhood Development in South Africa 2005-2010. 
intervention preschool programmes in Gauteng'. The objectives were threefold. The first objective was to describe the school readiness of children with hearing impairment upon graduation from the EHDI preschools. The second objective was to describe the schooling profile of children with hearing impairment upon completion of the foundation phase of formal schooling. The third objective was to describe the academic achievements of children with hearing impairment upon completion of their foundation phase of formal schooling.

\section{Methods and design Participants}

Eight children identified with hearing impairment and enrolled in two EHDI preschools were included in the study, along with their Grade 3 teachers. The children comprised four males and four females, ranging in age from 9.7 to 12.7 years, with a median age of 11.1 years. Six of the children were white and two were mixed race (Table 1). Four of the children were enrolled at EHDI preschool A, and the other four were enrolled at EHDI preschool B. Early Hearing Detection and Intervention preschool $\mathrm{A}$ is situated in Johannesburg and offers a specialised preschool programme for children with hearing impairments and/or language delay, with English as a medium of instruction, while EHDI preschool B is situated in Pretoria and offers habilitation services for children with hearing impairments in a preschool setting, with English and Afrikaans as medium of instruction (Maluleke, Khoza-Shangase \& Kanji 2019b).

The children had to meet the following criteria for their EHDI preschool records to be included in the study:

- Present with a bilateral, moderate hearing impairment or greater.

- Use amplification devices such as hearing aids and/or cochlear implants, in order to compensate for the hearing impairment.

Children who presented with co-morbidities such as cognitive impairment in addition to hearing impairment were excluded from the study.

\section{Procedure}

In order to identify potential participants for the study, the researchers obtained written consent from the two EHDI

TABLE 1: Demographic information.

\begin{tabular}{lclll}
\hline Participant no. & Age (years, months) & Gender & Race & Home language \\
\hline 1 & 9.9 & Female & Coloured & Afrikaans \\
2 & 10.8 & Male & White & English \\
3 & 11.7 & Male & White & English \\
4 & 10.9 & Female & White & English \\
5 & 9.7 & Male & White & English \\
6 & 12.7 & Female & White & Afrikaans \\
7 & 10.3 & Female & White & English \\
8 & 12.5 & Male & Coloured & Afrikaans \\
\hline
\end{tabular}

Source: Maluleke, N.P., Khoza-Shangase, K. \& Kanji, A., 2019b, 'Hearing impairment detection and intervention in children from centre-based early intervention programmes', Journal of Child Health Care 23(2), 232-241. https://doi.org/10.1177/1367493518788477 preschools, allowing the researchers access to the preschool records. Subsequently, the researchers compiled a list of caregivers' contact details for all potential children using non-probability sampling. Primary caregivers of all potential children were contacted by telephone, and permission was requested to review their child's preschool records and to invite their child's Grade 3 teacher to participate in the study. Consent forms were then emailed to the primary caregivers after they indicated their willingness to allow the researchers access to their child's preschool records. The record review of written and printed material was undertaken to gather information pertaining to (1) the child's hearing impairment, (2) ages at identification and initiation of early intervention services and (3) school readiness abilities at graduation from the EHDI preschool.

Thereafter, in order to gain access to the Grade 3 teacher participants, the researchers obtained written consent from the headmasters of the primary schools where the children completed Grade 3. Grade 3 teachers of all participants were contacted by telephone and invited to participate in the study. Consent forms were then emailed to teachers who agreed to participate after they indicated their willingness to partake in the research study. The Grade 3 teacher questionnaire, which was newly developed for the current study and was pre-tested on five primary school teachers who were not part of this study, was used. The selfadministered questionnaire, which was in English, comprised two sections: Section A related to the child's academic performance and Section B related to the child's general performance in the classroom.

\section{Data analysis}

Data were analysed using descriptive statistics. Univariate analysis was conducted in the study, according to two major characteristics: measures of dispersion and measures of central tendency (Salkind 2014). These included the mean, mode and range.

\section{Results}

Hearing impairment in all the children was identified late. These findings have been provided in detail in Maluleke et al. (2019b). Ages at identification ranged from 7 to 49 months. The mean age at identification was 27 months, and the median age was 24 months. The ages at the initiation of early intervention services ranged between 17 and 50 months. The mean age at the commencement of early intervention services was 29.9 months, and the median age was 24 months. The degree of the hearing impairment ranged from moderate to profound.

Upon graduation from the EHDI preschool, based on evidence from their assessment reports, one child presented with the above-average communication abilities, two children presented with spoken language abilities that approximated age-appropriate norms and five children presented with delayed communication abilities. 
TABLE 2: The children's 'school readiness' upon graduation from EHDI preschools.

\begin{tabular}{|c|c|c|c|c|c|c|c|c|}
\hline Expected school readiness skills and competencies & C1 & $\mathbf{C 2}$ & C3 & C4 & C5 & C6 & C7 & C8 \\
\hline Attention skills & No & No & Yes & Yes & Yes & Yes & Yes & Yes \\
\hline \multicolumn{9}{|l|}{ Concentrates on a task for at least $11 \mathrm{~min}$ ? } \\
\hline Spoken language abilities & No & No & No & Yes & No & Yes & Yes & Yes \\
\hline \multicolumn{9}{|l|}{ Has a command of the language? } \\
\hline \multicolumn{9}{|l|}{ Use sentences to express ideas and needs? } \\
\hline Mathematical concepts & No & No & Yes & Yes & Yes & Yes & Yes & Yes \\
\hline Counts up to at least $10 ?$ & & & & & & & & \\
\hline \multicolumn{9}{|c|}{ Understands the concept of counting, sorting and grouping? } \\
\hline \multicolumn{9}{|l|}{ Understands the concept of size } \\
\hline \multicolumn{9}{|l|}{ Writes numbers? } \\
\hline Early literacy & No & No & Yes & Yes & Yes & Yes & Yes & Yes \\
\hline \multicolumn{9}{|l|}{ Names basic colours? } \\
\hline \multicolumn{9}{|l|}{ Knows the letters of the alphabet? } \\
\hline \multicolumn{9}{|l|}{ Knows the name and sounds of letters? } \\
\hline
\end{tabular}

\section{School readiness}

The children's school readiness, published in Maluleke et al. (2019a), was obtained from the retrospective record review, reflect school readiness based on attention skills, spoken language abilities, mathematical concept knowledge and early literacy skills (Harrington et al. 2010). These findings are depicted in Table 2. A 'yes' indicates that the child presented with age-appropriate skills and competencies, while a 'no' indicates that the child's skills and competencies were not age appropriate.

Findings of this study revealed that only three children attained age-appropriate school readiness; the remaining five children did not attain age-appropriate school readiness as demonstrated by below-average attention, spoken language abilities, concept knowledge and early literacy abilities.

\section{Schooling profile}

The children's schooling profile was obtained from the Grade 3 Teacher questionnaires. At the end of Grade 3, participants were enrolled in three categories of schools. Four of the children were enrolled in mainstream schools, two in remedial schools and two in schools for Learners with Special Education Needs (LSEN).

The findings at the end of Grade 3, as depicted in Table 2, revealed progress in attention skills, spoken language abilities, mathematical concepts and early literacy when teachers' reports were compared to the record reviews as reported by Maluleke et al. (2019a).

The children enrolled in mainstream schools had attained ageappropriate school readiness upon graduation from the EHDI preschools, except for one. Child no. 4 (C4) had not attained age-appropriate school readiness and was subsequently enrolled in a remedial school upon graduation from the EHDI preschool. However, because of her excellent academic performance at the remedial school, it was recommended that she be enrolled in a mainstream school thereafter.

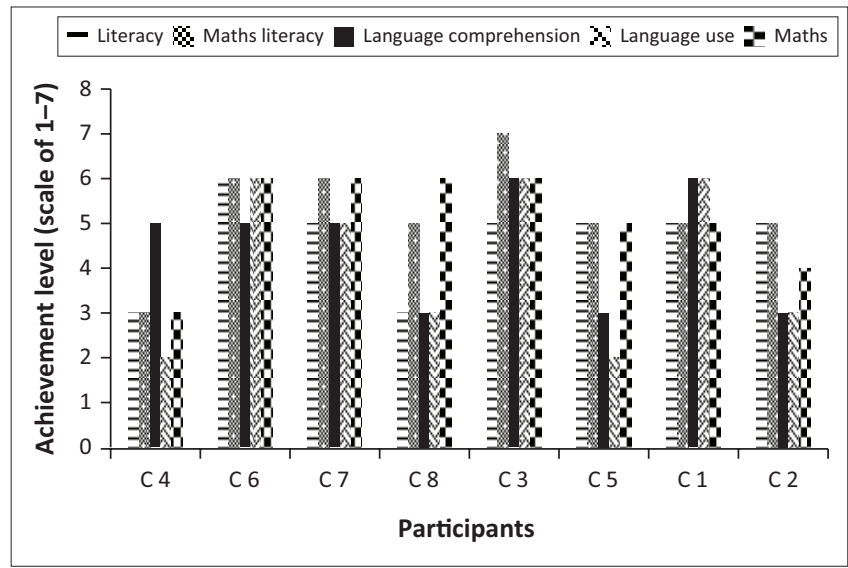

FIGURE 1: The children's academic performance upon completion of Grade 3.

All four of the children who were enrolled in either the remedial or LSEN schools had not attained age-appropriate school readiness. Of the two children enrolled in a school for LSEN, Child no.1 (C1) was enrolled in a school for the deaf where the medium of instruction was South African Sign Language. Child no. 2 (C2) was initially enrolled in a remedial school; however, because of his poor performance, it was recommended that he be enrolled in a school for LSEN thereafter.

\section{Academic achievement}

The children's academic achievement upon completion of Grade 3 was also obtained from the Grade 3 Teacher questionnaires. Results of the study demonstrate that all the children successfully completed Grade 3 (Figure 1).

Six of the eight children successfully completed the foundation phase in the prescribed 3 years. Only two children completed the foundation phase in 4 years, as they both repeated Grade 1. C4 repeated Grade 1 as she was initially enrolled in a remedial school, and it was recommended that she attend a mainstream school because of her excellent academic achievement. However, her mother insisted that she repeat Grade 1 in the mainstream school in order to 
ensure that she had 'obtained a good grounding'. C2 repeated Grade 1 as he was initially enrolled in a remedial school where he experienced academic difficulties and was subsequently enrolled in a school for LSEN.

No differences in achievement level were observed among the children according to the category of school they were enrolled in.

\section{Discussion}

Upon exit from EHDI preschools, only three children attained age-appropriate school readiness. The remaining five children did not attain age-appropriate school readiness as demonstrated by poor attention skills, spoken language abilities, concept knowledge and early literacy skills. The children who attained age-appropriate school readiness also presented with spoken language abilities that were age-appropriate or approximated age-appropriate norms. Despite the acknowledged small sample size in the current study, this finding supports the belief that spoken language abilities are crucial in the development of age-appropriate school readiness (Zaidman-Zait \& Young 2008). Children who have a solid foundation in spoken language abilities have the ability to apply their linguistic knowledge to concepts such as alphabet knowledge, colour recognition, number identification, time and sequence, which are part of the aspects of school readiness (Harrington et al. 2010). However, spoken language deficits in children with hearing impairment have a negative impact on their ability to master early literacy skills necessary for school readiness and academic achievement (Umat et al. 2018). This finding highlights the need for comprehensive EHDI programmes that will meet the unique needs of children with hearing impairment and their families, preventing (1) the poor educational attainment, (2) inequality and (3) socioeconomic challenges experienced by children with an unidentified or late-identified hearing impairment (Albino \& Berry 2013).

Once the children's school readiness was established, the study sought to describe the children's schooling profile at the end of the foundation phase. Results of the study indicate that children were enrolled in mainstream schools, remedial schools and LSEN schools. Four of the children were enrolled in mainstream schools, two in remedial schools and two in schools for LSEN. Children enrolled in mainstream schools presented with spoken language abilities that were ageappropriate or approximated age-appropriate norms. The current study's findings are consistent with the assertion that spoken language abilities were central in determining the 'appropriateness' of mainstream education for children with hearing impairment (Marschark \& Knoors 2013; Shaver et al. 2014), essentially it is the 'goodness-of-fit' between the child and the education environment (Harrington et al. 2010; Umat et al. 2018). This finding is positive, particularly for the South African context where there is a scarcity of schools that cater for children with hearing impairment. It also demonstrates that when children with hearing impairment are identified and managed appropriately, they are likely to join the mainstream school system (Butler et al. 2015), opening opportunities for them that evidence currently shows are limited.

The last objective of the study was to describe the academic achievement of children with hearing impairment at the end of the foundation phase. Six of the children completed the foundation phase in the prescribed 3 years. The remaining two children completed the foundation phase in 4 years, after repeating Grade 1. Both participants repeated Grade 1 because of inappropriate enrolment in remedial schools upon graduation from the EHDI preschools. This finding about academic achievement highlights the need for appropriate enrolment of children with a hearing impairment in the school environment that will enable them to reach their full potential (Harrington et al. 2010; Umat et al. 2018). Thus, school readiness results must be used as a guide to directly support children's developmental and academic achievement outcomes (Moreno 2013).

The reported finding also affirms the Committee on Children with Disabilities (1994) position that:

Early intervention of children with developmental disabilities leads to effective therapy of conditions for which definitive treatment is available. However, even in those instances in which the condition cannot be fully reversed, early intervention efforts improve children's outcomes and enables families to develop the strategies and obtain the resources for successful functioning. (p. 863)

Current findings support the established clear link between early identification and intervention of hearing impairment and speech and language development, social and emotional development, as well as scholastic outcomes (Khoza-Shangase \& Kanji 2021; Maluleke et al. 2019b; Sininger, Grimes \& Christensen 2010; Van Dyk, Swanepoel \& Hall 2015).

\section{Conclusion}

Current findings, which are preliminary in nature, demonstrate that through access to ECD services, such as EHDI programmes, children with hearing impairment are allowed to develop the school readiness required to experience academic success thus mitigating the risk of school failure, drop out and limited employment opportunities associated with an unidentified hearing impairment. Thus, there is a need for urgent attention by the South African Health, Social Development and Basic Education Departments to invest in initiatives aimed at properly resourcing, coordinating and managing ECD services, including EHDI programmes. Early Hearing Detection and Intervention programmes should ensure continuity of care to include the provision of services in school (Khoza-Shangase \& Kanji 2021; Khoza-Shangase et al. 2021). Such services should adhere to a collaborative approach involving families of children with hearing impairment, teachers and early intervention professionals. 


\section{Acknowledgements Competing interests}

The authors have declared that no competing interest exists.

\section{Authors' contributions}

All authors contributed equally to the research and writing of this article.

\section{Ethical considerations}

All procedures performed in the study University's Human Research Ethics Committee-Medical (Protocol Number: M130240). Furthermore, the work adhered to the Helsinki Declaration of 1975, as revised in 2008.

\section{Funding information}

This research received no specific grant from any funding agency in the public, commercial or not-for-profit sectors.

\section{Data availability}

Data are available from the corresponding author, N.P.M., upon reasonable request.

\section{Disclaimer}

The views and opinions expressed in this article are those of the authors and do not necessarily reflect the official policy or position of any affiliated agency of the authors.

\section{References}

Albino, N. \& Berry, L., 2013, 'Early childhood development services in South Africa: What are the next steps?', in South African child gauge, pp. 78-81, viewed 25 July 2019, from https://open.uct.ac.za/bitstream/handle/11427/3896/Cl_chapters sachildgauge13 EcdNextSteps 2013.pdf?sequence $=1$.

Britto, P.R., Rana, A.J., Pasic, M. \& Mannathoko, C., 2012, School radiness and transition, New UNICEF, York, viewed 10 June 2019, from https://www. oxfordshire.gov.uk/sites/default/files/file/ear;y-years-childcare/ schoolradinessandtransitionunicef.pdf

Brown, J.M., 2016, Developing a parenting skills programme to enhance the school readiness of Grade $R$ learners in resource poor communities, viewed 02 March 2020, from http://dspace.nwu.ac.za/bitstream/handle/10394/24991/Brown JM_2016.pdf?isAllowed=y\&sequence $=1$.

Butler, I.R.T., Ceronio, D., Swart, T. \& Joubert, G., 2015, 'Age of diagnosis of congenital hearing loss: Private vs, public healthcare sector', South African Medical Journal 105(11), 927-929. https://doi.org/10.7196/SAMJ.2015.v105i11.9576

Clelland, M.M.M.C. et al., 2015, 'Developmnt and self-regulation', Handbook of Child Psychology and Developmental Science, in W.F. Ovrton, P.C.M. Molenaar \& R.M. Psychology and Developmental Science, in W.F. Ovrton, P.C.M. Molenaar \& R.M. (7th Edition), John Wiley \& Sons, Inc., New Jersey. https://doi.org.10.1002/ (7th Edition), John Wiley \&
9781118963418.childpsy114

Cole, P., Armstrong, L. \& Pemberton, C., 2010, 'The role of language in the development of emotion and regulation', in S.D. Calkins \& M.A. Bell (eds.), Child development at the intersection of emotion and cognition, pp. 59-77, American Psychology the intersection of emotion

Darling-Hammond, L., Flook, L., Cook-Harvey, C., Barron, B. \& Osher, D., 2020 Implications for educational practice of the science of learning and development', Applied Developmental Science 24(2), 97-140. https://doi.org/10.1080/1088869 1.2018.1537791

Department of Education, 1995, White paper on education and training, Department of Education, Republic of South Africa, viewed 03 March 2019, from https://www. gov.za/documents/white-paper-education-and-training.

Department of Education, 2001, White paper 5 on early childhood education, Department of Education, Republic of South Africa, viewed 04 March 2019, from https://www.gov.za/sites/default/fils/gcis_document/201409/educ1791.pdf.

Department of Welfare, 1997, White paper for social welfare, Department of Welfare, Republic of South Africa, viewed 03 March 2019, from http://www.default/files/ gcis_document/201409/whitepaperonsocialwelfar0.pdf.
De Villiers, D., 2010, 'Deaf education in South Africa', American Annuls for the Deaf 155(4), 493-946. https://doi.org/10.1353/aad.2010.0038

Developmnt and self-regulation. Handbook of Child Psychology and Developmental Science, in W.F. Ovrton, P.C.M. Molenaar \& R.M. Lerner, Handbook of child psychology and developmental science (7th Edition), John Wiley \& Sons, Inc., New Jersey. https://doi.org.10.1002/9781118963418.childpsy114.

EHDI, 2018, 'Professional board for speech, language and hearing professions early hearing detection and intervention programmes in South Africa position statement', Children, viewed 06 June 2019, from http://www.hpcsa.co.za/Uploads/SLH/ Guidelines\%20for\%20Early_Hearing_Detection_and_Inervention_(EHDI)_2018.pdf.

Hammer, C., Morgan, P., Farkas, G., Hillemeier, M., Bitetti, D. \& Maczuga, S., 2017, 'Late talkers: A population-based study of risk factors and school readiness consequnces', Journal of Speech, Language, and Hearing Research 60(3), 607626. https://doi.org/10.1044/2016_JSLHR-L-15-0417

Harrington, M., Desjardin, J.L. \& Shea, L.C., 2010, 'Relationships between early child factors and school readiness skills in young children with hearing loss', Communication Disorders Quarterly 32(1), 50-62. https://doi.org/10.1177/1525740109348790

Institute of Medicine (IOM) and National Research Council (NRC), 2015, Transforming the workforce for children Birth through Age 8: A unifying foundation, The National Academies Press, Washington, DC.

JClH, Muse, C., Harrison, J., Yoshinaga-Itano, C., Grimes, A., Brookhouser, P.E. et al., 2013, 'Supplement to the JCIH 2007 position statement: Principles and guidelines for early intervention after confirmation that a child is deaf or hard of hearing', Pediatrics 131(4), e1324-e1349. https://doi.org/10.1542/peds.2013-0008

Khoza-Shangase, K., 2021, 'Confronting realities to early hearing detection in South Africa', in K. Khoza-Shangase \& A. Kanji (eds.), Early detection and intervention in audiology: An African perspective, p. 66, Wits University Press, Johannsburg.

Khoza-Shangase, K. \& Kanji, A., 2021, 'Best practice in South Africa for early hearing detection and intervention', in K. Khoza-Shangase \& A. Kanji (eds.), Early detection and intervention in audiology: An African perspective, p. 264, Wits University Press, Johannsburg.

Khoza-Shangase, K., Sebothoma, B. \& Moroe, N., 2021, 'Tele-audiology as part of efforts to enhance inclusivity and equality through ICT', in M. Maguvhe, R.S. Mphahlele \& S. Moonsamy (eds.), Empowering students and maximising inclusiveness and equality through ICT, pp. 223-243, Brill.

Lang-Roth, R., 2014, 'Hearing impairment and language delay in infants: Diagnostik and genetic', Laryngo-Rhino-Otologie 93(Suppl 1), S126-S149. https://doi. org/10.1055/s-0033-1363214

Maluleke, N.P., Khoza-Shangase, K. \& Kanji, A., 2019a, 'Communication and schoo readiness abilities of children with hearing impairment in South Africa: $A$ retrospective review of early intervention preschool record', South African Journa of Communication Disorders 66(1), 1-7. https://doi.org/10.4102/sajcd.v66i1.604

Maluleke, N.P., Khoza-Shangase, K. \& Kanji, A., 2019b, 'Hearing impairment detection and intervention in children from centre-based early intervention programmes', Journal of Child Health Care 23(2), 232-241. https://doi.org/10.1177/136749 3518788477

Maluleke, N.P., Khoza-Shangase, K. \& Kanji, A., 2021, 'An integrative review of current practice models and/or process of family-centered early intervention for children who are deaf or hard of hearing', Family \& Community Health 44(1), 59-71. https://doi.org/10.1097/FCH.0000000000000276

Marschark, M. \& Knoors, H., 2013, 'Educating deaf children: Language, cognition and learning', Deafness and Education International 14(3), 136-160. https://doi.org/1 0.1179/1557069X12Y.0000000010

Marti, M., Merz, E.C., Repka, K.R., Landers, C., Noble, K.G. \& Duch, H., 2018, 'Parent involvement in the getting ready for school intervention is associated with changes in school readiness skills', Frontiers in Psychology 9, 759. https://doi. org/10.3389/fpsyg.2018.00759

McClelland, M.M.M, Geldhof, G.J., Cameron, C.E. \& Wanless, S.B., 2015, 'Developmnt and self-regulation, Handbook of Child Psychology and Developmental Science', in W.F. Overton, P.C.M. Molenaar \& R.M. Lerner (eds.), Handbook of child psychology and developmental science (7th Edition), John Wiley \& Sons, Inc., New Jersey. https://doi.org.10.1002/9781118963418.childpsy114

Medina, R. \& Suthers, D.D., 2008. 'Bringing representational practice from log to light', ICLS'08: Proceedings of the 8th international conference on conference for the learning science-volume 2. https://doi.org/10.5555/1599871.1599879

Menting, B., Van Lier, P. \& Koot, H., 2010, 'Language skill, peer rejection, and the development of externalising behaviour from kindergarten to fourth grade Journal of Child Psychology and Psychiatrry and Allied Disciplines 52(1), 72-79. https://doi.org/10.1111/j.1469-7610.2010.02279.x

Moeller, M. \& Tomblin, J., 2015, 'An introduction to the outcoms of children with hearing loss study', Ear and Hearing 36(1), 4S-13S. https://doi.org/10.1097/ AUD.0000000000000210

Moodley, S. \& Storbeck, C., 2015, 'Narrative review of EHDI in South Africa', The South African journal of communication disorders = Die Suid-Afrikaanse tydskrif vir Kommunikasieafwykings 62(1), E1-E10. https://doi.org/10.4102/sajcd.v62i1.126

Moreno, M.A., 2013, 'School readiness', JAMA Pediatrics 167(8), 784. https://doi. org/10.1001/jamapediatrics.2013.2959

Mukani, S., Ling, L. \& Ghani, H., 2007, 'Educational performance of pediatric cochlear implant recipients in mainstream classes', International Journal of Pediatric Otorhinolaryngology 71(2), 231-240. https://doi.org/10.1016/j.ijporl.2006. 10.005

National Research Council, Institute of Medicine, Board on Children, Youth \& Families, 2015, Transforming the workforce for children birth through age 8: A unifying foundation, in L. Allen \& B. Kelly (eds.), The National Academies Press. 
Packer, L., 2018, 'How hearing loss affects school performance', Healthy Hearing viewed 04 June 2020, from https://www.healthyhearing.com/report/52433-Howhearing-loss-affects-school-perfo
nginpoorschoolperformance.

Salkind, N., 2014, Statistics for people who (think they) hate statistics, 5th edn., Sage, Sage Publishing, Inc., Thousand Oaks.

Shaver, D.M., Marschark, M., Newman, L. \& Marder, C., 2014, 'Who is where? Characteristics of deaf and hard-of-hearing students in regular and special schools', Journal of Deaf Studies and Deaf Education 19(2), 204-219. https://doi. org/10.1093/deafed/ent056

Sininger, Y.S., Grimes, A. \& Christensen, E., 2010, 'Auditory development in early amplified children: Factors influencing auditory-based communication outcomes in children with hearing loss', Ear and Hearing 31(2), 166-185. https://doi. org/10.1097/AUD.0b013e3181c8e7b6

Storbeck, C. \& Moodley, S., 2011, 'ECD policies in South Africa - What about children with disabilities?', Journal of African Studies and Development 3, 1-8, viewed 14 September 2020, from http://hihopes.co.za/Images/StorbeckandMoodleyECD\%5CnPolicy.pdf.

Swanepoel, D.W., 2009, 'Early detection of infant hearing loss in South Africa', South African Medical Journal 99(3), 158-159.
Tinajero, A.R., 2010, Scaling-up early child development in Cuba: Cuba's Educate Your Child Program - strategies and lessons from the expansion process, viewed 06 June 2020 from https://www.early-child-development-in-cuba-cubas-educateyour-child-program-strategies-and-lessons-from-the-expansion-process/

Umat, C., Mukari, S.Z.-M.S., Nordin, N., Annamalay, T.A.L. \& Othman, B.F., 2018, 'Mainstream school readiness skills of a group of young cochler implant users', International Journal of Pediatric Otorhinolaryngology 107, 69-74. https://doi. org/10.1016/j.ijporl.2018.01.031

Van Dyk, M., Swanepoel, D. \& Hall, J., 2015, 'Outcomes with OAE and AABR screening in the first $48 \mathrm{~h}$ - Implications for newborn hearing screening in developing countries', International Journal of Pediatric Otorhinolaryngology 79(7), 1034-1040. https://doi.org/10.1016/j.ijporl.2015.04.021

Viviers, A., Biersteker, L. \& Moruane, S., 2013, 'Strengthening ECD service delivery Addressing systemic challenges', in South African Child Gauge, pp. 34-43, viewed 14 September 2020, from https://www.ci.uct.ac.za/sites/default/files/ images/367/Child-Gauge/South-Africa-Child-Gaug-2013/Gauge2013Systems.pdf.

Zaidman-Zait, A. \& Young, R.A., 2008, 'Parental involvement in the habilitation process following children's cochlear implantation: An action theory perspective', Journa of Deaf Studies and Deaf Education 13(1), 39-60. https://doi.org/10.1093/ deafed/enm051 